\title{
FRECUENCIA Y ASPECTOS EPIDEMIOLÓGICOS DEL PARASITISMO INTESTINAL EN ESTUDIANTES DE 5 A 16 AÑOS DE LEIMEBAMBA, AMAZONAS-PERÚ.
}

\author{
FREQUENCY AND EPIDEMIOLOGICAL ASPECTS OF INTESTINAL PARASITISM \\ AMONE STUDENTS IN THE AGES OF 5-16 IN LEIMEBAMBA, AMAZONAS - PERU
}

${ }^{1}$ Christian Rivera-Salazar ${ }^{\mathrm{a}},{ }^{1}$ Cristian Polo-Zavala,${ }^{1} \mathrm{Juan}$ Pedro-Huaman ${ }^{\mathrm{b}}$

\section{RESUMEN}

Se determinó la frecuencia del parasitismo intestinal y su relación con el sexo, la edad y el tipo de agua de consumo de escolares de cinco a dieciséis años de la ciudad de Leimebamba, Amazonas-Perú, entre noviembre del 2010 y febrero del 2011.

La muestra estuvo conformada por 215 escolares elegidos al azar. Los análisis coproparasitoscópicos se efectuaron en una muestra fecal por niño a través de la técnica de examen directo.

La frecuencia de parasitados fue de $64.2 \%$ y se halló 5 especies de parásitos intestinales, entre protozoarios y helmintos, siendo su frecuencia Giardia lamblia (37.7\%), Entamoeba coli (30.4\%), Ascaris lumbricoides (26\%). Trichuris trichiura (4.3\%) e Hymenolepis. nana(1.5\%).

En la población estudiantil de Leimebamba, el parasitismo por protozoarios y helmintos intestinales está relacionado al grupo de edad entre 5 y 9 años e ingerir agua de mala calidad.

Palabras clave: parasitismo intestinal, aspectos epidemiológicos, protozoarios y helmintos.

\section{ABSTRACT}

The frequency of intestinal parasites and its relationship to gender, age and type of drinking water for five to sixteen years students from Leimebamba City, Amazonas-Peru, between November 2010 and February 2011 were determined.

The sample consisted of 215 randomly selected schools. The coproparasitoscopic analyzes were performed in a fecal sample per child through direct examination.

Parasitized frequency was $64.2 \%$ and 5 species of intestinal parasites were found between protozoa and helminths, and its frequency Giardia lamblia (37.7\%), Entamoeba coli (30.4\%), Ascaris lumbricoides (26\%). Trichuris trichuria (4.3\%) and Hymenolepis. nana (1.5\%).

In the student population from Leimebamba, parasitism by protozoa and helminths is related to age group between 5 and 9 years and to poor drinking water.

Keywords. intestinal parasitism, epidemiological, protozoa and helminths

\footnotetext{
${ }^{1}$ Universidad Nacional de Jaén. Cajamarca.

Biólogo - Microbiólogo.

${ }^{\mathrm{b}}$ Biólogo.
} 


\section{I.-INTRODUCCIÓN}

La frecuencia mundial de las distintas parasitosis intestinales es alta, en especial en zonas geográficas donde las condiciones ecológicas favorecen la persistencia de los parásitos, además de las características socioeconómicas poblaciones como la pobreza, ignorancia y deficiente infraestructura; factores que comparten los países en vías de desarrollo y que, lamentablemente, en América Latina no han presentado modificaciones importantes en los últimos 50 años. (Sanchez, y otros 2000). En general tienen baja mortalidad, pero igualmente ocasionan importantes problemas sanitarios y sociales debido a/su sintomatología y complicaciones (Acuña, y otros 1999).

Las enteroparasitosis pueden transcurrir durante largo tiempo asintomáticas; pero también pueden llegar a provocar cuadros digestivos, inclusive con severa repercusión sobre el crecimiento y desarrollo de los niños (Calchi, y otros 1996; Levav, y otros 1995; Páez, y otros 1994; Rivero, y otros 1997; Simoes, y otros 2000). Se está investigando la incidencia que pueden tener las infecciones parasitarias intestinales sobre el rendimiento escolar; por ejemplo a través de la irritabilidad y el cansancio que provocan, con repercusión sobre la capacidad intelectual y la atención (Sanchez, y otros 2000)

Las parasitosis intestinales se consideran un problema de salud pública que afecta a individuos de todas las edades y sexos (Atias, y otros 1991; Ramos, y otros 1997); pero se presentan sobre todo en los primeros años de vida, ya que este grupo de población aún no ha adquirido los hábitos higiénicos necesarios para prevenirlas y no se ha desarrollado inmunidad frente a los diferentes tipos de parásitos (Calchi, y otros 1996; Páez, y otros 1994; Simoes, y otros. 2000).

Estas infecciones se producen en el hombre cuando sus hábitos y costumbres se interrelacionan con los ciclos de vida de los helmintos y protozoarios agentes causales de las mismas, que pueden tomar diversa ubicación en el organismo humano, causando transtornos clínicos aparentes o no (Atias, y otros1991; Díaz, y otros 2000; Rivero, y otros 2000).

Entre las causas de morbilidad a nivel mundial, la producida por parásitos intestinales se sitúa en el tercer lugar, precedida por las infecciones respiratorias agudas y las diarreas, siendo la prevalencia de los principales helmintos: Ascaris lumbricoides 1.250 .000 de los casos, Ancylostomideos 990.000 y Trichuris trichiura 700.000 de casos (Canese, y otros 1999).

En América Latina la presencia, frecuencia e incidencia de parásitos en diferentes regiones, es un muy buen indicador del estado de salud de la población. (Atias 1991).

Las infecciones por protozoarios y helmintos intestinales en diferentes zonas de la región yunga oriental peruana presentan elevadas prevalencias ; así por ejemplo, en el distrito Juan Guerra (Tarapoto, departamento de San Martín) $84,2 \%$ de escolares presentan Blastocystis hominis (Concha, y otros1997); en Bagua (departamento de Amazonas), 45,1\% de la población examinada está infectada con Giardia lamblia (Pareja, y otros 2000); en Puerto Maldonado (departamento de Madre de Dios) $72,6 \%$ de los pobladores esta parasitado por anquilostomideos, 59,7\% con Ascaris lumbricoides e igual cifra por Trichuris trichiura (Valdivia, y otros 2000); y en Rodríguez de Mendoza (departamento de Amazonas) el parasitismo por $A$. lumbrioides en escolares fue del orden de 51,7\%5. (Ibáñez,y otros 2001).

El objetivo del presente trabajo es determinar la frecuencia de infección por protozoarios y helmintos intestinales y su asociación con el tipo de consumo de agua, sexo y edad en la población escolar del distrito de Leimebamba, en la Provincia de Chachapoyas, Amazonas, Perú. 


\section{II.-MATERIAL Y METODOS}

Éste estudio observacional, descriptivo y transversal fue ejecutado entre noviembre de 2010 y febrero de 2011 en zona Nor- Oriental del territorio peruano, en el espacio de transición entre la cordillera andina y la llanura Amazónica;, en el distrito de Leimebamba, departamento de Amazonas.

Se efectuó análisis Coproparasitològicos, uno por individuo, de 215 escolares de ambos sexos, de 5 a 16 años. La recolección de muestras fecales, fue tomada por conveniencia (porque no se tenía un número aproximado de escolares).

Todos los escolares que participaron en el estudio eran aparentemente sanos, habían concurrido habitualmente a la escuela y carecían de agua potable. Adicionalmente a sus quehaceres estudiantiles, la mayoría de escolares ayudan a sus padres en labores agrícolas.

Antes de efectuar los exámenes parasitológicos se impartieron charlas informativas que tuvieron la finalidad de explicar la importancia y el motivo de la investigación, así también, la manera de recolectar la muestra fecal y llenar la ficha individual con datos epidemiológicos de utilidad para la investigación, como: sexo, edad y tipo de agua de consumo.

\section{ANÁLISIS PARASITOLÓGICOS}

Las muestras fecales, una por individuo, fueron recolectadas en vasos descartables nuevos y trasladados al laboratorio para su análisis correspondiente. En el laboratorio, cada muestra fecal fue procesada por el método directo con el fin de observar presencia de formas evolutivas móviles de parásitos de tamaño microscópico (trofozoítos, quistes de protozoos: Entamoeba histolytica, Giardia lamblia, Balantidium coli, etc.; así como larvas o huevos de helmintos: Strongyloides stercoralis, Ancylostoma duodenale, Necator americanus, Trichostrongylus sp., Paragonimus, Fasciola, etc.).

\section{TRATAMIENTO ESTADÍSTICO}

Las frecuencias halladas fueron sometidas a la prueba de Chi Cuadrado, con un límite de confiabilidad del 95\% ( $\mathrm{P}=0.05 \%)$, afín de determinar la probable asociación entre el parasitismo intestinal y los factores epidemiológicos considerados. (Steel y cols. 1980)

\section{III.-RESULTADO}

En las 215 muestras examinadas que corresponden a otros tantos escolares se encontró $64.2 \%$ de parasitados (Tabla 1) y se hallaron 5 especies distintas de parásitos intestinales, entre protozoarios y helmintos, de ellas Giardia lamblia y Entamoeba coli fueron los protozoarios con mayor frecuencia Ascaris lumbricoides fue el helminto con mayor frecuencia con $26.1 \%$ y el de menor frecuencia Himenolepis nana con $1.5 \%$ (Tabla 2).

Cuando se relacionó la frecuencia de infección de parásitos intestinales en relación con el grupo de edad, se encontró que tanto protozoos como helmintos intestinales se presentan con frecuencias mayores en escolares de 5 a 9 años (Tabla 3).

La frecuencia de infección de parásitos intestinales en relación con el sexo, esta se distribuye en proporción uniforme (Tabla 4).

Finalmente la frecuencia de infección de parásitos intestinales en relación al tipo de consumo de agua se presentó en mayor frecuencia el consumo de agua no segura. (Tabla 5).

TABLA 1. Frecuencia de parasitados y no parasitados, en estudiantes de 5 a 16 años en el distrito de Leimebamba, Provincia de Chachapoyas, Amazonas, noviembre del 2010 y febrero del 2011. 


\begin{tabular}{lcccc}
\hline CONDICIÔN & \multicolumn{2}{c}{ Parasitados } & \multicolumn{2}{c}{ No Parasitados } \\
\hline \multirow{2}{*}{ Total } & Número & Porcentaje & Número & Porcentaje \\
& 138 & 64.2 & 77 & 35.2 \\
\hline
\end{tabular}

TABLA 2. Frecuencia de Parásitos Intestinales, en estudiantes de 5 a 16 años en el distrito de Leimebamba, Provincia de Chachapoyas, Amazonas, noviembre del 2010 y febrero del 2011.

\begin{tabular}{llc}
\hline Parásito Intestinal & & Protozoario \\
\hline Giardia lamblia & 52 & Porcentaje \\
Entamoeba coli & 42 & 37.7 \\
& & 30.4 \\
Ascaris lumbricoides & 36 & Helminto \\
Trichuris trichiura & 6 & 26.1 \\
Himenolepis nana & 2 & 4.3 \\
Total & 138 & 1.5 \\
\hline Se examinaron 215 estudiantes & 100 \\
\hline
\end{tabular}

TABLA 3.Frecuencia de Parásitos intestinales, en relación con el grupo de edad, en el distrito de Leimebamba, Provincia de Chachapoyas, Amazonas, entre noviembre del 2010 y febrero del2011.

\begin{tabular}{|c|c|c|c|c|c|c|}
\hline \multirow{3}{*}{ Parasito Intestinal } & \multicolumn{4}{|c|}{ Grupo de edad } & \multirow{2}{*}{\multicolumn{2}{|c|}{ Total }} \\
\hline & \multicolumn{2}{|c|}{$5-9$} & \multicolumn{2}{|c|}{$10-16$} & & \\
\hline & $\mathrm{N}^{\circ}$ & $\%$ & $\mathrm{~N}^{\circ}$ & $\%$ & $\mathrm{~N}^{\circ}$ & $\%$ \\
\hline Giardia lamblia & 32 & 23.1 & 20 & 14.5 & 52 & 37.6 \\
\hline Entamoeba coli & 30 & 21.7 & 12 & 8.7 & 42 & 30.4 \\
\hline Ascaris lumbricoides & 30 & 21.7 & 6 & 4.3 & 36 & 26.3 \\
\hline Trichuris trichiura & 4 & 2.9 & 2 & 1.4 & 6 & 4.3 \\
\hline Himenolepis nana & 1 & 0.7 & 1 & 0.7 & 2 & 1.4 \\
\hline Total & 97 & 70.3 & 41 & 29.7 & 138 & 100 \\
\hline
\end{tabular}

TABLA 4.Frecuencia de Infección de Parásitos intestinales, en relación al sexo, en el distrito de Leimebamba, Provincia de Chachapoyas, Amazonas, entre noviembre del 2010 y febrero del2011.

\begin{tabular}{lccccccc}
\hline \multirow{2}{*}{ Parasito Intestinal } & \multicolumn{4}{c}{ Sexo } & \multicolumn{3}{c}{ Total } \\
\cline { 2 - 5 } & \multicolumn{2}{c}{ Femenino } & \multicolumn{2}{c}{ Masculino } & & \multirow{2}{*}{ No } \\
\cline { 2 - 5 } & $\mathrm{N}^{\circ}$ & $\%$ & $\mathrm{~N}^{\circ}$ & $\%$ & & $\%$ \\
\hline Giardia lamblia & 28 & 20.3 & 24 & 17.4 & 52 & 37.7 \\
Entamoeba coli & 22 & 15.9 & 20 & 14.5 & 42 & 30.4 \\
Ascaris lumbricoides & 21 & 15.2 & 15 & 10.9 & 36 & 26.1 \\
Trichuris trichiura & 3 & 2.1 & 3 & 0.3 & 6 & 4.3 \\
Himenolepis nana & 1 & 0.7 & 1 & 0.1 & 2 & 1.4 \\
Total & 75 & 54.3 & 63 & 45.7 & 138 & 100 \\
\hline
\end{tabular}

TABLA 5. Frecuencia de Infección de Parásitos intestinales, en relación al tipo de consumo de agua, en el distrito de Leimebamba, Provincia de Chachapoyas, Amazonas, entre noviembre del 2010 y febrero del 2011.

\begin{tabular}{|c|c|c|c|c|c|c|}
\hline \multirow[t]{3}{*}{ Parasito Intestinal } & \multicolumn{4}{|c|}{ Tipo de agua de consumo } & & \\
\hline & \multicolumn{2}{|c|}{$\begin{array}{l}\text { Agua } \\
\text { segura }\end{array}$} & \multicolumn{2}{|c|}{$\begin{array}{l}\text { Agua no } \\
\text { segura }\end{array}$} & \multicolumn{2}{|c|}{ Total } \\
\hline & $\mathrm{N}^{\circ}$ & $\%$ & $\mathrm{~N}^{\circ}$ & $\%$ & $\mathrm{~N}^{\circ}$ & $\%$ \\
\hline Giardia lamblia & 2 & 1.4 & 50 & 36.2 & 52 & 37.7 \\
\hline Entamoeba coli & 4 & 2.9 & 38 & 27.5 & 42 & 30,4 \\
\hline Ascaris lumbricoides & 3 & 2.2 & 33 & 23.9 & 36 & 26.1 \\
\hline Trichuris trichiuria & 0 & 0 & 6 & 4.4 & 6 & 4.4 \\
\hline Himenolepis nana & 0 & 0 & 2 & 1.4 & 2 & 1.4 \\
\hline Total & 9 & 6.5 & 129 & 93.5 & 138 & 100 \\
\hline
\end{tabular}

\section{IV.-DISCUSION}

En la presente investigación se halló que un $62 \%$ de niños tienen infección por parásitos. Las razones de este resultado están referidas, básicamente, a condiciones ambientales y al comportamiento humano. En el primer caso influye directamente el tipo de clima templado y lluvioso de la zona que condiciona la presencia de un suelo arcilloso, siempre húmedo, óptimo para la evolución, mantenimiento y propagación de los helmintos y protozoarios.etc. En relación al comportamiento, tienen poco conocimiento de medidas higiénicas y salubridad pública lo que hace que la disposición de excretas, la preparación de los alimentos y el cuidado para no infectarse por parásitos intestinales sean deficientes (Apt 1987).

La presencia de quistes de Giardia lamblia en alto porcentaje (37.7\%), es mayor que en otras áreas geográficas de clima templado y lluvioso como: Juan Guerra (22.4\%) en Tarapoto (Concha, y otros 1997), el 18.5\% detectado en pobladores de Puerto Maldonado (Valdivia, y otro 2000), el 22.3 encontrado en niños de Tarapoto (Murga, y otros 2000) y al 23,3\% informado en la comunidad nativa de Cañanbuque, San Martin (Garaycochea, y otros 2002). Esto se explicaría a las malas condiciones de saneamiento ambiental presentes en la zona (deficiente eliminación de basuras y excretas, la pululación de las 
moscas, los grados de contaminación fecal del agua de bebida y riego, con la subsecuente contaminación de alimentos)constituyendo los principales factores de mantención y diseminación de la giardiasis.(Atias 1991).

La elevada frecuencia $(30.4 \%)$ con que se presenta $E$. coli, es menor al $57.4 \%$ encontrado en escolares de Juan Guerra, en Tarapoto (Concha y cols., 1997) y al 58\% detectado en una población rural de San Martin (Garaycohea y cols., 2002), sin embargo es preciso mencionar que resulta ser uno de los protozoarios intestinales más encontrados junto a G. lamblia, esto se debería al fecalismo infantil permisible en la población infantil por los malos hábitos higiénicos.

Los geohelmintos encontrados son: $A$. lumbricoides, T. trichiura e H. nana, esto se explicaría por los precarios hábitos higiénicos y por el suelo húmedo por largos periodos, convirtiéndose en un ambiente óptimo para la evolución, mantenimiento y propagación de estos helmintos.

Cuando se relacionó la edad con las frecuencias parasitadas halladas, se encontró que había una distribución mayor en niños entre 5 a 9 años de edad, esto se explicaría a que en este grupo, el nivel educacional e intelectual en relación a enfermedades parasitarias es deficiente con relación a los escolares de 10 a 16 años.

Como en la mayoría de trabajos previos, se encontró independencia entre las frecuencias halladas y el sexo, esto podría explicarse, que el tubo digestivo tiene la misma conformación en hombres y mujeres, porque los hábitos alimenticios son similares en ambos y también las oportunidades de infectarse con protozoarios y helmintos intestinales (Apt 1987).

En relación a la relación existente entre las frecuencias halladas y el tipo de consumo de agua, se detalla dependencia, esto debido a que en la zona, es común el uso de agua contaminada con heces, para bebida y riego de terrenos agrícolas, con la subsecuente contaminación de los alimentos, constituyéndose uno de los principales factores de riesgo de mantención y diseminación de las parasitosis.

\section{V.-CONCLUSIONES}

Existe un elevada frecuencia de parásitos intestinales $(64.2 \%)$ con una elevada prevalencia de protozoarios $(68.1 \%)$ y mayor que los helmintos (31.9\%).

Los principales factores relacionados a las infecciones por parásitos intestinales fueron pertenecer al grupo de edad entre 5 y 9 años e ingerir agua de mala calidad

La relación entre el sexo y la frecuencia de parasitosis intestinal, es independiente.

Los protozoarios intestinales más frecuentes son G. lamblia con $37.7 \%$ y E. coli con $30.4 \%$

El helminto más frecuente es $A$. lumbricoides con $26.1 \%$

\section{VI.-REFERENCIAS BIBLIOGRAFICAS}

Acuña, A.; Da Rosa, D.; Colombo, H.; Saur, S.; Alfonso, A.; Combolo, A.; Castelló, R. y Zanetta, E, 1999; Parasitosis Intestinales en Guarderías comunitarias de Montevideo. Rev. Med. Urug. 1999; 15:5-12p.

Apt W. 1987; Helmintiasis intestinales humanas en América Latina, prevalencia actual y sus factores contribuyentes. Parasitología 11(4):155-166p.

Atias, A. 1991; Parasitología Clínica. Tercera Edición. Santiago de Chile. Publicaciones técnicas Mediterráneas LTDA. 168 p.

Calchi, M.; Chourio, G. y Díaz, I. 1996; Helmintiasis Intestinal en niños de una comunidad marginal del Municipio Maracaibo. Estado Zulia-Venezuela. Kasmera. 24: 17-38p.

Canese, A.; Barrios. E.; Castro, L. y Canese, J. 1999; Prevalencia de Parásitos Intestinales encontrados en niños en Paraguay. Rev. Parag de Microb. 19(1). 
Concha R, Huiza A, Espinoza Y, Sevilla C, Lazo B. 1997; Estudio parasitológico en escolares de cinco centros educativos del Distrito Juan Guerra, Provincia de Tarapoto. Bol Peru Parasitol 12(1): 10.

Garaycochea MC, Mori S, Velásquez S. 2002. Parasitismo intestinal e intensidad de infección en una población rural de San Martín. En: V Congreso Peruano de Parasitología. Trujillo: Asociación Peruana de Parasitología; 61p.

Ibáñez N, Jara C. 2001; Nueva área de paragonimiosis: Chota, Cajamarca, Perú. Rev Peru Parasitol 15(1):36-41.

Ibañez N, Jara C, Guerra A, Diaz E.2001. Prevalencia del Enteroparasitismo en escolares de comunidades nativas del Alto Marañón, Amazonas, Perú. Rev.Peru Parasitol. vol 02.

Levav, M.; Mirsky, A.; Schantz, P.; Castro, S. and Cruz, M. 1995; Parasitic infection in malnourisned school children: efeccts on behaviour and EEG. Parasitology. 1: 103-110.

Murga N, Ruiz P. 2000; Parásitos intestinales asociados a enfermedad diarreica aguda en menores de dos años de Tarapoto. En: IV Congreso Peruano de Parasitología. Lima: Asociación Peruana de Parasitología; 98p.

Páez, B. y Calchi, M. 1994; Prevalencia de Parasitosis Intestinales en alumnos del preescolar INSP José Celestino Azuage, el Policiíta“. Municipio Maracaibo. Estado Zulia. Kasmera. 22: 51-69p.

Pareja M, Zamora P. 2000;Parasitosis en pobladores de la ciudad de Bagua. Enero '99-Dic '99. En: IV Congreso Peruano de Parasitología. Lima: Asociación Peruana de Parasitología.69 p.

Perú, 1983. Ministerio de Salud. II Seminario Taller: Vigilancia, prevalencia y control de las infecciones parasitarias intestinales. Lima: Instituto Nacional de Salud (INS). 84p.
Ramos, L. y Salazar, R. 1997; Infestación Parasitaria en niños de Cariaco - Edo. Sucre, Venezuela y su relación con las condiciones económicas. Kasmera. 25: 175-189.

Rivero, Z.; Chango, Y. e Iriarte, H.1997; Enteroparasitosis en alumnos de la Escuela Básica "Dr. Jesús Maria Portillo" del Municipio Maracaibo - Estado Zulia. Kasmera. 25: 121$139 \mathrm{p}$.

Sánchez J, Tay J, Guerrero L, Romero R, Ruíz D y Rivas C. Mayo-Junio, 2000; Frecuencia de parasitosis intestinales en asentamientos humanos irregulares, Rev Fac Med UNAM Vol.43 No.3 .68p.

Simoes, M.; Rivero, Z.; Carreño, G.; Lugo, M.; Maldonado, A.; Chacín, I.; Parra, M.; Méndez, Y. y Marquina, M. Prevalencia de enteroparasitosis en una Escuela Urbana en el Municipio San Francisco, Estado Zulia, Venezuela.

Steel R, Torrie JH.1980. ;Bioestadística: Principios y procedimientos. 2da ed. Cali, Colombia: Edit Mc Graw-Hill.

Valdivia L, Córdova E, Liu M, Neira M, Vásquez L, Ayaqui R, et al. El parasitismo intestinal familiar e intensidad de las infecciones por geohelmintos en Puerto Maldonado. 1998. En: IV Congreso Peruano de Parasitología. Lima: Asociación Peruana de Parasitología; 2000.93 p.

\section{CORRESPONDENCIA}

Christian Rivera Salazar

PasajeAltamirano \#121 - Jaén, Cajamarca, Perú xyian26@hotmail.com 